\title{
Basophil Count, Percentage of Basophil and Basophil Lymphocyte Ratio in Psychiatry Practice: Current Approaches and Future Directions
}

\author{
Mehmet Hamdi Örüm ${ }^{1}$ \\ ${ }^{1}$ Kahta State Hospital, Psychiatric Outpatient Clinic, Adiyaman, Turkey
}

Dear Editor;

Basophils, the least abundant granulocytes, have poorly understood functions. They contain histamine and express the affinity immunoglobulin E ( $\mathrm{lgE}$ ) receptor FceRla and may play a role in protective $\mathrm{T}$ helper 2 (Th) cytokine-mediated immunity to some parasites. Also, basophils are thought to contribute to the pathogenesis of allergic diseases/reactions, asthma, and acute or chronic myelogenous leukaemia. ${ }^{[1,2]}$ The basophil count (BASO), percentage of basophil (BASO\%) and basophil lymphocyte ratio (BLR) are derived from complete blood count (CBC) and may lead to some interpretation.

Recent studies revealed that $\mathrm{CBC}$ can give important clues about the acute and chronic stages of diseases. ${ }^{[3]}$ Various studies have also been reported on psychiatric disorders such as major depressive disorder, ${ }^{[4,5]}$ alcohol use disorder ${ }^{[6]}$ and opioid use disorder, ${ }^{[3]}$ etc. Researchers examining the relationship between depression and anxiety and inflammation suggested that white blood cell (WBC) subset fraction and counts may provide indirect evidence of an altered inflammatory system in anxious depression. Baek et al. ${ }^{[7]}$ demonstrated that anxious depression can lead to decreased BASO\%. However, Kara et al. ${ }^{[5]}$ found no significant difference in basophil-related parameters and BLR in suicide attempts that could be associated with depression and/ or anxiety. Orum et al. ${ }^{[3]}$ reported no association between basophil and opioid use, whereas BLR correlated with alcohol use duration. ${ }^{[6]}$

As with many studies on CBC parameters, basophil-related studies have many limitations. Firstly, the retrospective nature of these studies is the most important limitation. Longitudinal studies are needed to see how basophil behaves during acute, chronic stages of the diseases, before and after treatments. The known smoking effect that changes blood values is a confounding factor. The life style and nutritional characteristics of the individuals are also other confounding factors. On the other hand, it is known that basophil survives for a very short time in the body and can be affected by many different conditions. Nowadays, CBC parameters such as basophil in psychiatric disorders are mostly interpreted within the framework of acute inflammatory events. However, basophils have a memory response, and perhaps the values we have identified in the disease may reflect chronic events. ${ }^{[3]}$ The ability of basophils to recognize and to react to antigen suggests that they may be involved in the development of memory immune responses. Allergic conditions have been reported to occur more frequently in developmental disorders. Strom and Silverberg ${ }^{[8]}$ reported that paediatric eczema may be associated with increased risk of speech disorder. For these reasons, basophil suggests a possible association between developmental psychiatric disorders and previous infections. [9] This hypothesis needs more detailed studies. However, basophil-related parameters and BLR in such psychiatric disorders have not yet been studied.

In conclusion, it is suggested that studies examining the relationship between basophil-related parameters and psychiatric disorders should be directed from the acute inflammatory response topic to the chronic events due to its feature of memory response. The main subjects that can be investigated for this purpose are developmental stuttering, tic disorder and obsessive compulsive disorder. 


\section{REFERENCES}

1. Denzel A, Maus UA, Rodriguez Gomez M, et al. Basophils enhance immunological memory responses. Nat Immunol 2008; 9(7):73342.

2. Parlakpinar $\mathrm{H}$, Orum $\mathrm{MH}$, Sagir M. Pathophysiology of myocardial ischemia reperfusion injury: A review. Med Science 2013;2(4):93554.

3. Orum $M H$, Kara MZ, Egilmez OB, Kalenderoglu A. Complete blood count alterations due to the opioid use: what about the lymphocyte-related ratios, especially in monocyte to lymphocyte ratio and platelet to lymphocyte ratio? J Immunoassay Immunochem 2018;14:1-12.

4. Orum $\mathrm{MH}$, Kara MZ, Egilmez OB. Mean platelet volume and neutrophil to lymphocyte ratio as parameters to indicate the severity of suicide attempt. J Immunoassay Immunochem 2018; 39(6):647-59.

5. Kara MZ, Orum MH, Egilmez OB. Relationship between immune cells and violent/nonviolent suicide attempts and controls: What about the lymphocyte-related ratios and neutrophil-related parameters? Kaohsiung J Med Sci 2019;35(5):315-6.

6. Orum $\mathrm{MH}$, Kara MZ, Egilmez OB. Relationship between immune cells and alcohol dependents and controls: what about the lymphocyte-related ratios? J Immunoassay Immunochem 2018; 39(3):348-50.

7. Baek JH, Kim HJ, Fava M, et al. Reduced venous blood basophil count and anxious depression in patients with major depressive disorder. Psychiatry Investig 2016;13(3):321-6.

8. Strom MA, Silverberg Jl. Eczema is associated with childhood speech disorder: A retrospective analysis from the National Survey of Children's Health and the National Health Interview Survey. Journal of Pediatrics 2016;168:185-92.

9. Kara $M Z$, Örüm $M H$, Sekmen E. Is there a relationship between basophil, platelet-related parameters and developmental stuttering? Anadolu Psikiyatri Dergisi 2019;21(2):187-94. (doi: 10.5455/apd.57722) 